\title{
Evolution of reproductive systems in filamentous ascomycetes. I. Evolution of mating types
}

\author{
M. J. NAUTA \& R. F. HOEKSTRA \\ Department of Genetics, Agricultural University, Dreyenlaan 2, 6703 HA Wageningen, The Netherlands
}

\begin{abstract}
In the ascomycete family of Sordariaceae both heterothallism (with two mating types) and homothallism (without mating types) are common. A population genetic model is made in an attempt to find out under which conditions evolution from one system to the other is conceivable. Analysis shows that evolution from hetero- to homothallism is possible but evolution from homo- to heterothallism is improbable. As in these haploid fungi self-fertilization has other consequences than in diploid organisms, homothallism seems to have little disadvantage. It is found that polymorphism in homo- and heterothallism can be stable, although this has not yet been found in Sordariaceae in nature.
\end{abstract}

Keywords: ascomycetes, evolution, heterothallism, homothallism, mating types, reproduction.

\section{Introduction}

Most population genetic models about the evolution of sex and mating systems concern animals and plants (e.g. Maynard Smith, 1978; Bell, 1982; Stearns, 1987; Michod \& Levin, 1988). The fungi are largely overlooked. Some of the reasons for this may be the relative lack of knowledge about their population structure and genetics, the complex life cycle of many fungi and the puzzling variety in reproductive systems. This variation, however, also offers an opportunity for comparative studies of the evolutionary forces that shape the different mating systems.

This study presents a model of the evolution of mating types in filamentous ascomycetes, exemplified by the family Sordariaceae. This family includes some genetically well-known species, e.g. Neurospora crassa, Podospora anserina and Sordaria fimicola, which live on rotten plant material or herbivore dung. They show relatively simple life cycles (see below). Some population genetic (Perkins \& Turner, 1988) and molecular (Glass et al., 1990) data are also available and provide useful information. The model will probably also be valid for many other ascomycete species, but these are not treated explicitly here.

In the Sordariaceae (as in many other ascomycete families) roughly two mating systems exist: homothallism and heterothallism. Homothallic species are self fertile and have no mating types, whereas hetero- thallic species are self-sterile and possess mating types. Here 'mating types' is defined as 'two different sexes without morphological sex-differentiation'. These mating types receive different names in different species but are called + and - in this study.

Note that the terms monoecy and dioecy are confusing in this context. In plants these terms refer to species in which individuals produce gametes of only one sex or of both sexes. All Sordariaceae make both, independent of mating type. [The implication of this will be discussed in a subsequent paper (M. J. Nauta \& R. F. Hoekstra, 1992).]

A remarkable phenomenon is the occurrence of both homo- and heterothallic species within many related ascomycete genera and families. This means that homo- and/or heterothallism must have evolved independently quite often. One may suspect, then, that the threshold for switching from one system to another cannot be too high.

The purpose of this study is to discover the conditions, defined in general fitness parameters under which homothallism can evolve to heterothallism and vice versa.

\section{The model}

The model is based on a typical Sordariaceae life cycle as presented in Fig. 1. Note the following characteristics. 


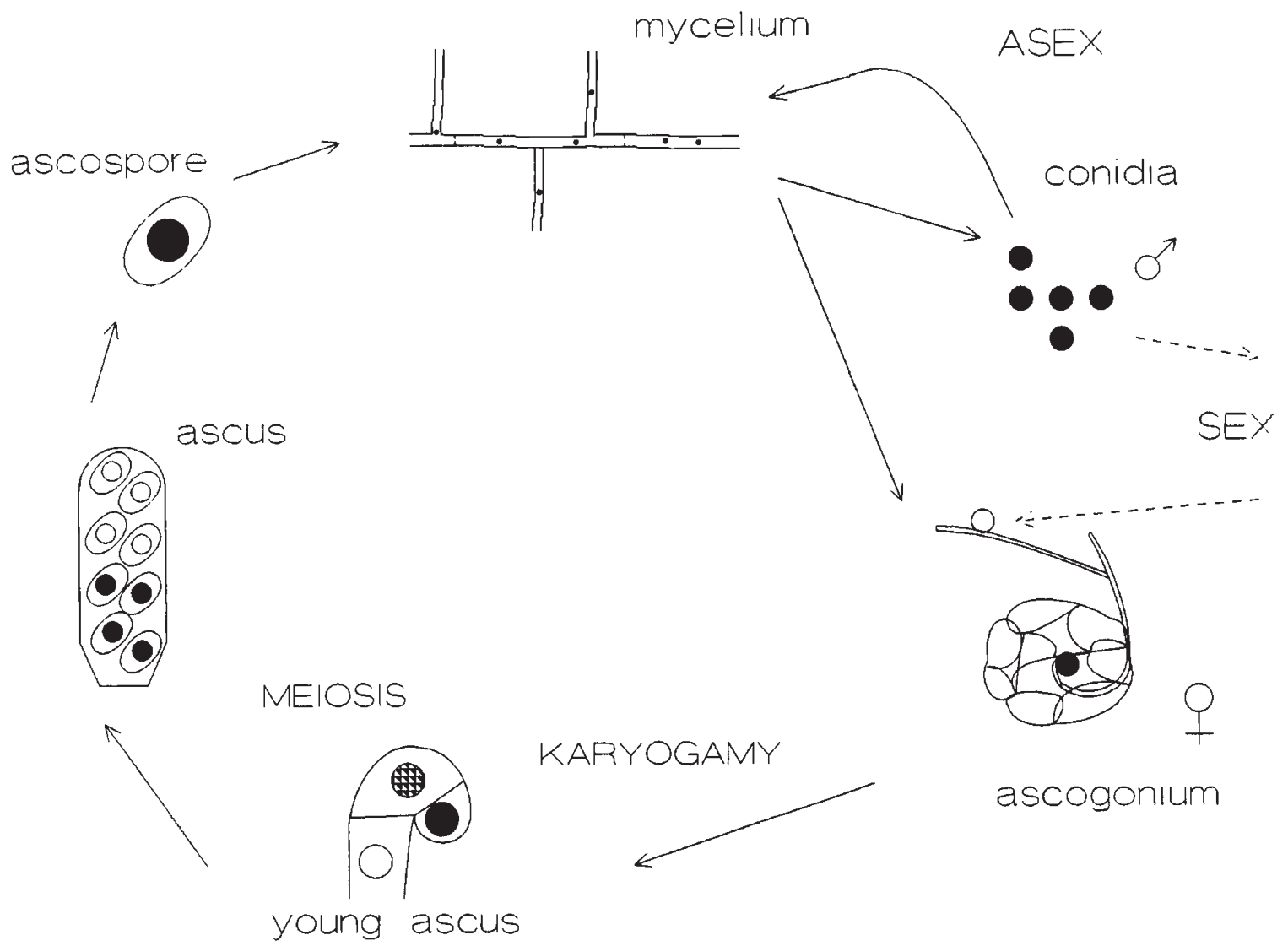

Fig. 1 Life cycle of the heterothallic model organism. A haploid mycelium contains either nuclei of mating type $+(\bullet)$ or of mating type $-(0)$. The conidia can develop asexually into a new mycelium or fertilize ascogonia of the opposite mating type. After karyogamy and meiosis an ascus with eight ascospores (four of each mating type) is formed. The homothallic model organism has the same life cycle, but has no mating types (self-fertilization is possible).

1 The life cycle is haploid. There is only a very short stage of diploidy (in the young ascus) which is immediately followed by meiosis.

2 Each individual mycelium forms both conidia and ascogonia, that is both male and female gametes. As stated above this is completely independent of mating type.

3 The conidia serve as both male gametes and asexual spores. [This is a simplification of the situation found in $N$. crassa, where micro- as well as macroconidia exist. The first seem to serve mainly as a fertilizing agent and the second as an asexual spore (Perkins \& Turner, 1988). In the laboratory, however, both can perform the two functions.]

4 Because of haploidy self-fertilization does not imply recombination. [A similar phenomenon in ferns is called intragametophytic selfing (Klekowski, 1979; Hedrick, 1987).] From a genetic point of view then the formation of selfed spores is equivalent to forming asexual spores.

Furthermore, the following assumptions are made in the model.
There are two heterothallic mating types $(+$ and -$)$ with frequencies $x_{1}$ and $x_{2}$, and one homothallic 'mating type' \pm with frequency $x_{3}\left(x_{1}+x_{2}+x_{3}=1\right)$. These three types are assumed to be determined by three alleles at one locus. The fitness of a heterothallic cross $+x-$ equals 1 , the crosses $\pm x-$ and $\pm x+$ have a fitness $w_{1}\left(w_{1} \leq 1\right)$. The homothallic crossing $\pm \times \pm$ has a fitness $w_{2}$ when it concerns outcrossing (frequency $1-s$ ) and $w_{3}$ when selfing (frequency $s$ ).

All mycelia produce the same amount of ascogonia and conidia. There is an excess of conidia formed, so all ascogonia are fertilized. [This can be compared with ovules and pollen in higher plants (Charlesworth \& Charlesworth, 1978).]

The conidia disperse randomly over the area. Some land on unoccupied substrate and have a chance to germinate. Others land on a mycelium and are able to fertilize ascogonia. There is an active attraction between unlike mating types as in, for example, Podospora anserina (Esser, 1959) and Bombardia lunata (Zickler, 1952). Identical mating types do not attract each other. This means that both a + conidium landing 
on a +mycelium, and a - conidium landing on a - mycelium will get lost. All ascogonia on a heterothallic mycelium will be fertilized, but of course not by conidia of its own mating type.

Both sexual spores (ascospores) and asexual spores (conidia) are formed. The difference in fitness between these spores (relating to differences in viability as well as frequency) is expressed in the parameter $\Theta$, denoting the fitness of an ascospore relative to that of a conidium. As $\Theta$ will appear of no importance in the analysis of this model, its precise definition will be discussed in a subsequent paper (M. J. Nauta and R. F. Hoekstra, 1992).

Taking all these assumptions into account the following recurrence relations can be derived:

$$
\begin{aligned}
& W x_{1}^{\prime}=x_{1}\left[1+\frac{1}{2} \Theta\left(\frac{x_{2}+w_{1} x_{3}}{x_{2}+x_{3}}+\frac{x_{2}}{x_{1}+x_{3}}+w_{1}(1-s) x_{3}\right)\right] \\
& W x_{2}^{\prime}=x_{2}\left[1+\frac{1}{2} \Theta\left(\frac{x_{1}+w_{1} x_{3}}{x_{1}+x_{3}}+\frac{x_{1}}{x_{2}+x_{3}}+w_{1}(1-s) x_{3}\right)\right] \\
& W x_{3}^{\prime}=x_{3}\left[1+\frac{1}{2} \Theta\left\{(1-s)\left[2 w_{2} x_{3}+w_{1}\left(x_{1}+x_{2}\right)\right]\right.\right. \\
& \left.\left.+\frac{w_{1} x_{2}}{x_{1}+x_{3}}+\frac{w_{1} x_{1}}{x_{2}+x_{3}}+2 w_{3} s\right\}\right]
\end{aligned}
$$

where

$$
\begin{aligned}
W= & 1+\Theta\left\{x_{3}(1-s)\left[w_{1}\left(x_{1}+x_{2}\right)+w_{2} x_{3}\right]+x_{2} \frac{x_{1}+w_{1} x_{3}}{x_{2}+x_{3}}\right. \\
& \left.+x_{1} \frac{x_{2}+w_{1} x_{3}}{x_{1}+x_{3}}+w_{3} s x_{3}\right\}
\end{aligned}
$$

When $x_{1}=x_{2}$ (which can be shown to be the case in all equilibrium conditions, see Appendix), with $x_{1}+x_{2}=p$ and $x_{3}=q$, this can be simplified to

$$
\begin{aligned}
& W p^{\prime}=p\left\{1+\frac{1}{2} \Theta\left[w_{1}(1-s) q+\frac{p+w_{1} q}{\frac{1}{2} p+q}\right]\right\} \\
& W q^{\prime}=q\left\{1+\frac{1}{2} \Theta\left[(1-s)\left(2 w_{2} q+w_{1} p\right)+\frac{w_{1} p}{\frac{1}{2} p+q}+2 w_{3} s\right]\right\}
\end{aligned}
$$

where

$$
W=1+\Theta\left[q(1-s)\left(w_{1} p+w_{2} q\right)+p \frac{\frac{1}{2} p+w_{1} q}{\frac{1}{2} p+q}+w_{3} s q\right] .
$$

When $(2 b)$ is rewritten as

$\frac{q^{\prime}}{q}=\frac{F(q)}{W}$,

it is easy to see that $q$ will increase when $f(q)=F(q)-W>0$.

It can be deduced that $f(q)=A \cdot q^{2}+B \cdot q+C$, with

$A=(1-s)\left(w_{2}-w_{1}\right)$,

$B=1+w_{2}(1-s)+w_{3} s-\frac{1}{2} w_{1}(5-s)$,

$C=\frac{1}{2} w_{1}(3-s)+w_{3} s-1$

There will be an equilibrium when $f(q)=0$, so two equilibrium points can be deduced, one stable

$\hat{q}_{1}=-\frac{-B-\sqrt{ }\left(B^{2}-4 A C\right)}{2 A}$,

and one unstable

$\hat{q}_{2}=\frac{-B+\sqrt{\left(B^{2}-4 A C\right)}}{2 A}$

Using the Taylor expansion it can be shown that for $p \approx 0$ : heterothallism can invade if $A+B+C<0$, that is if

$w_{1}>2 \frac{w_{2}(1-s)+w_{3} s}{2-s}$

and for $q \approx 0$ : homothallism can invade if $C>0$, that is if

$w_{1}>2 \frac{1-w_{3} s}{3-s}$

There appear to be four possible states: homothallism (ho), heterothallism (he), stable polymorphism (po) and a frequency-dependent state (fd), where neither type can invade (heterothallism being stable if $q>\hat{q}_{2}$, and homothallism if $\left.q<\hat{q}_{2}\right)$. Conditions (5) and (6) describe which of these states is reached, except in cases when $0<\hat{q}_{1}, \hat{q}_{2}<1$ where the final state depends on $q$. The four states occur in the following cases (for all $q$, except when stated otherwise). 
po: $(C>0$ and $A+B+C<0)$ or $(C>0$ and $A+B+C>0$ and $A>-\frac{1}{2} B>0$ and $\left.q<\hat{q}_{2}\right)$ or $(C<0$ and $A+B+C<0$ and $A<-\frac{1}{2} B<0$ and $q>\hat{q}_{2}$ ),

fd: $C<0$ and $A+B+C>0$,

he: $C<0$ and $A+B+C<0$ and $\operatorname{not}\left(A<-\frac{1}{2} B<0\right.$ and $\left.q>\hat{q}_{2}\right)$,

ho: $C>0$ and $A+B+C>0$ and $\operatorname{not}\left(A>-\frac{1}{2} B>0\right.$ and $\left.q<\hat{q}_{2}\right)$.

Before discussing some special cases, note that the invasion of heterothallism in a homothallic population is unlikely to happen with two mating types (two simultaneous mutations) at once. That is, one has to consider the introduction of one mating type first. This means that $x_{1}=0$ or $x_{2}=0$. Elaborating this case gives condition (5) again for heterothallic invasion. The frequency $q$ must be 0.5 at least, because the \pm mating type must also serve as a heterothallic partner.

The expressions $A, B$ and $C$ in formulae (3) and (4)

(a)

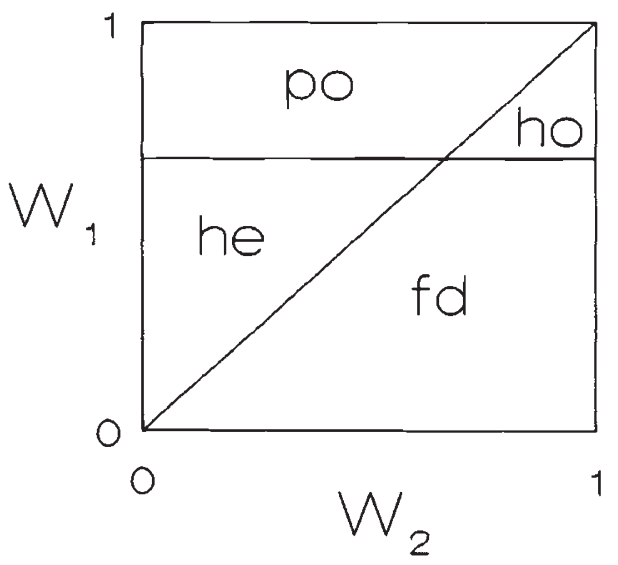

(c)

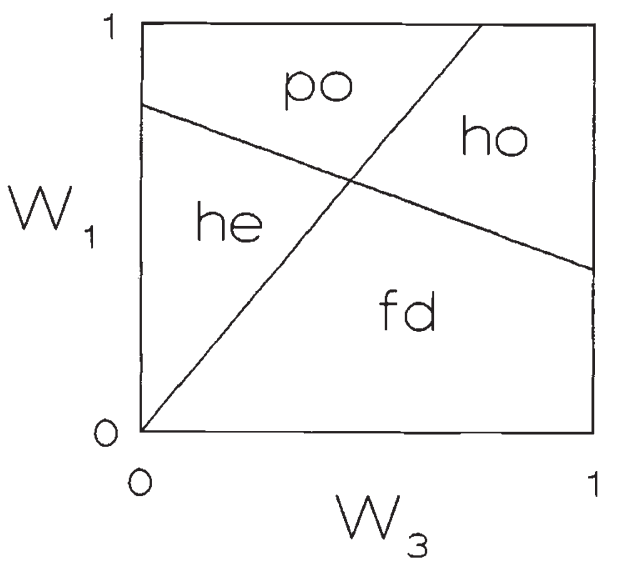

now become

$A=2(1-s)\left(w_{2}-w_{1}\right)$,

$B=2 s w_{3}-(1+s) w_{2}$,

$C=w_{1}$.

After the right mutation the second mating type can invade under the same conditions as the first [condition (5)]. To achieve a better impression of these formulae, some special cases will be considered. (See Fig. 2 for illustrations.)

1 No selfing: $s=0$

Homothallism can invade if $w_{1}>2 / 3$ and heterothallism if $w_{2}<w_{1}$.

2 All homothallics are selfing: $s=1$

Homothallism can invade if $w_{1}>1-w_{3}$ and heterothallism if $w_{1}>2 w_{3}$. (b)

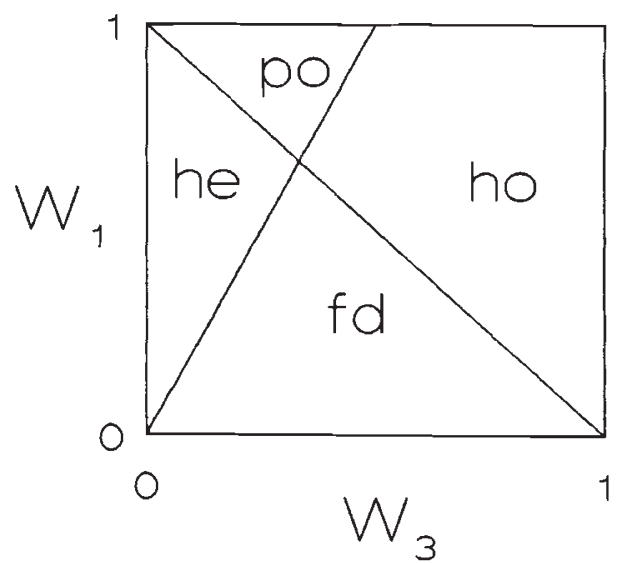

(d)

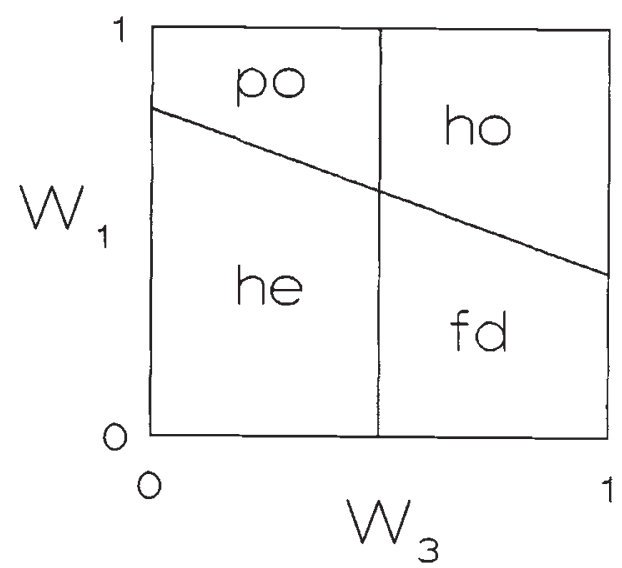

Fig. 2 Equilibrium states in four different cases. po $=$ stable polymorphism, he $=$ heterothallism, ho $=$ homothallism, $\mathrm{fd}=$ frequency dependent. (a) $s=0$ (no selfing, discussed as case 1 in the main text), (b) $s=1$ (all homothallics are selfing, case 2), (c) $s=0.5$ and $w_{2}=w_{3}$ (homothallic crosses are equally fit), (d) $s=0.5$ and $w_{1}=w_{2}$ (outbreeding crosses with homothallics are equally fit). 
3 All outcrossing sex has the same fitness: $w_{1}=w_{2}=1$

(a) selfing mildly deleterious: $w_{3} \geq 0.5$. Heterothallism can never invade, homothallism is stable.

(b) selfing strongly deleterious: $w_{3}<0.5$. Both homo- and heterothallism can invade, polymorphism is stable.

It is clear that conditions for homothallism to invade a heterothallic population will be much more easily realized than conditions for heterothallism to invade. A heterothallic population can only be stable with strong selection pressure against homothallism and/or selfing.

The model seems to suggest, therefore, that evolution from hetero- to homothallism may be possible but that evolution from homo- to heterothallism is expected to be rare. At the same time it shows that, when evolution from homo- to heterothallism or vice versa occurs, one should also expect to find populations polymorphic for this trait.

\section{Discussion}

One of the few discussions on the evolution of heterothallism in ascomycetes has been given by Olive (1958, 1963). He assumes that in the early evolution of the fungi, homothallism preceded heterothallism. In the homothallic species Sordaria fimicola, a number of mutations that affect the sexual process have been found. In the laboratory 'heterothallism' could be created by selecting for self-sterile colonies that could be crossed with each other (El Ani \& Olive, 1962). It is suggested that heterothallism might evolve by 'the occurrence and association of pseudo allelic selfsterility mutations in a compound locus of two or more subunits'.

The nature of the selective forces that promote the evolution of heterothallism under natural conditions is not clear but, according to Raper (1968) and Esser (1971, 1974), these should mainly be the promotion of outbreeding and the prevention of inbreeding. This last argument, however, deserves a closer look. What is meant by inbreeding is actually intragametophytic selfing. (Selfing as it occurs in diploids, intergametophytic selfing, is possible with both homo- and heterothallism in these fungi.) This selfing does not imply any recombination and is in fact equivalent to asexual reproduction. This means that the 'usual' disadvantages of inbreeding are not applicable here.

A model on the evolution of mating types in isogamous populations has been studied by Hoekstra (1982, 1987). Using comparable parameters (and with $s=0$ ), he found that heterothallism can invade if $w_{1}>w_{2}$ and homothallism if $w_{1}>1 / 2$. The difference with the present model is the second condition, which is less severe here $\left(w_{1}>2 / 3\right)$. The reason for this is the gamete differentiation in the present model and the fact that no ascogonia are lost by incompatible fusions.

It does not mean, however, that the evolution of heterothallism has become easier. It is hard to find convincing reasons why $w_{1}, w_{2}$ and/or $w_{3}$ should be considerably smaller than 1 .

The idea that heterothallism must have preceded homothallism in evolution is supported by DNA sequencing of the mating type genes of Neurospora crassa and the comparison of these sequences with other Sordariaceae (Glass et al., 1990; Metzenberg \& Glass, 1990). It has been found that + and - (called $A$ and a in Neurospora) are dissimilar and that most (but not all) homothallic species carry homologous sequences of both mating types in one haploid genome. Mating type switching, as in yeast (Herskowitz, 1988), is improbable in the Sordariaceae.

The fact that most homothallic Sordariaceae do not form conidia is not reflected in the model assumptions (Perkins \& Turner, 1988), which means that outcrossing can only take place via occasional mycelial contact. Thus in homothallic species, in nature, the frequency of selfing must be close to 100 per cent. This is supported by the finding that RFLP and mating-type analysis show far less variation within homothallic species than within heterothallic species (Glass et al., 1990).

This possible lack of conidia may make the model less valid for the evolution of homo- to heterothallism. It is easy to see, however, that a more realistic model would put a heterothallic mutant at an even greater disadvantage than in the present model because it decreases the chance of finding an appropriate mate. This will therefore only produce more severe conditions for the evolution of heterothallism.

The only explanation for the existence of heterothallic Sordariaceae seems to be that in some cases the fitness thresholds for intermediate stages are too high. More ecological research on these species is needed to find out if and why this should be the case.

Note that the model suggests that polymorphism in homo- and heterothallism may very well be stable. No report of such polymorphism has been found in Sordariaceae. In some species of comparative ascomycete families, both homo- and heterothallic strains are described [e.g. Glomerella cingulata (Wheeler, 1954), Gibberella zeae and Nectria haematococca (Booth, 1971)], but the stability of these strains in nature is somewhat obscure. It may well be that polymorphism occurs in some species but has never been reported.

The lack of conidia in many homothallic species, the differentiation in male and female gametes in addition to 
mating types, and the existence of related fungi imperfecti, offer some intriguing additional questions. These will be analysed in a comparative model (M. J. Nauta and R. F. Hoekstra, 1992).

\section{Acknowledgements}

These investigations were supported by the Foundation for Biological Research (BION), which is subsidized by the Netherlands Organization for Scientific Research (NWO; grant no. 811-439-085).

\section{References}

BELL, G. 1982. The Masterpiece of Nature: The Evolution and Genetics of Sexuality. University of Columbia Press, Berkeley.

воотн, C. 1971. The Genus Fusarium. Commonwealth Mycological Institute, Kew.

CHARLESWORTH, D. AND CHARLESWORTH, B. 1978. Population genetics of partial male-sterility and the evolution of monoecy and dioecy. Heredity, 41, 137-153.

EL ANI, A. S. AND OLIVE, L. S. 1962. The induction of balanced heterothallism in Sordaria fimicola. Proc. Natl. Acad. Sci., U.S.A., 48, 17-19.

ESSER, K. 1959. Die Incompatibilitätsbeziehungen zwischen geographischen Rassen von Podospora anserina (Ces.) Rehm. II. Die Wirkungsweise der Semi-IncompatibilitätsGene. Z. Vererbungsl., 90, 29-52.

ESSER, K. 1971. Breeding systems in fungi and their significance for genetic recombination. Mol. Gen. Genet., 110 , 86-100.

ESSER, K. 1974. Breeding systems and evolution. In: Carlile, M. J. and Skehel, J. J. (eds) Evolution in the Microbial World. Proceedings of the 24th Symposium of the Society of Genetics and Microbiology, Cambridge University Press, Cambridge, pp. 87-104.

GLASS, N. L., METZENBERG, R. L. AND RAJU, N. B. 1990. Homothallic Sordariaceae from nature: The absence of strains containing only the a mating type sequence. Exp. Mycol., 14, 274-289.

HEDRICK, P. w. 1987. Population genetics of intragametophytic selfing. Evolution, 41, 137-144.

HERSKOWITZ, I. 1988. Life cycle of the budding yeast Saccharomyces cerevisiae. Microb. Rev., 52, 536-553.

HOEKSTRA, R. F. 1982. On the asymmetry of sex: Evolution of mating types in isogamous populations. J. Theor. Biol., 98, 427-451.

HoEKSTRA, R. F. 1987. The evolution of sexes. In: Stearns, S. C. (ed.) The Evolution of Sex and its Consequences, Birkhauser Verlag, Basel, pp. 59-91.

KLEKOWSKI, E. J. 1979. The genetics and reproductive biology of ferns. In: Dyer, A. F. (ed.) The Experimental Biology of Ferns, Academic Press, London, pp. 133-170.

MARYNARD SMITH, J. 1978. The Evolution of Sex. Cambridge University Press, Cambridge.
METZENBERG, R. L. AND GLASS, N. L. 1990. Mating type and mating strategies in Neurospora. Bioessays, 12, 53-59.

MICHOD, R. E. AND LEVIN, B. R. 1988. The Evolution of Sex: An Examination of Current Ideas, Sinauer Associates Inc., Sunderland.

NAUTA, M. J. AND HOEKSTRA, R. F. 1992. Evolution of reproductive systems in filamentous ascomycetes. II. Evolution of hermaphroditism and other reproductive strategies. Heredity, 68, (in press).

OLIVE, L. S. 1958. On the evolution of heterothallism in fungi. Am. Nat., 42, 233-251.

OLIVE, L. S. 1963. Genetics of homothallic fungi. Mycologia, 55, 93-103.

PERKINS, D. D. AND TURNER, B. C. 1988. Neurospora from natural populations: Toward the population biology of a haploid eukaryote. Exp. Mycol., 12, 91-131.

RAPER, J. R. 1968. On the evolution of fungi. In: Ainsworth, G. C. and Sussman, A. S. (eds) The Fungi. An Advanced Treatise, Vol. III, Academic Press, New York, pp. 677-694.

STEARns, s. c. 1987. The Evolution of Sex and its Consequences, Birkhauser Verlag, Basel.

WHEELER, H. E. 1954. Genetics and the evolution of heterothallism in Glomerella. Phytopathology, 44, 342-345.

ZICKLER, H. 1952. Zur Entwicklungsgeschichte des Askomyzeten Bombardia lunata Zckl. Arch. Protistenk., 98, 1-70.

\section{Appendix}

\section{Equal heterothallic frequencies are stable}

Formulae (1a) and (1b) can be rewritten to

$\frac{x_{1}^{\prime}}{x_{2}^{\prime}}=\frac{x_{1}\left(a+b x_{2}\right)}{x_{2}\left(a+b x_{1}\right)}$

with

$a=\left[1+\frac{1}{2} \Theta w_{1}(1-s) x_{3}\right]\left(1-x_{1}\right)\left(1-x_{2}\right)+\frac{1}{2} \Theta w_{1} x_{3}$,

$b=\frac{i}{2} \Theta\left[1+x_{3}\left(1-w_{1}\right)\right]$.

It is easy to see that $x_{1}$ and $x_{2}$ are mutually interchangeable, so if $x_{1} / x_{2}$ converges to 1 for $x_{1}<x_{2}$, it will also converge to 1 for $x_{2}<x_{1}$.

If $x_{1}<x_{2}$, then $x_{1} / x_{2}$ converges to 1 without oscillations if

$\frac{x_{2}}{x_{1}}>\frac{a+b x_{2}}{a+b x_{1}}>1$

As both $a>0$ and $b>0$, these conditions hold if $x_{1}<x_{2}$. So $x_{1} / x_{2}$ always converges to 1 and equal frequencies of the heterothallic genotypes are stable. 